\title{
An IOT-enabled System for Marine Data Acquisition and Cartography
}

\author{
Rabab Al-Zaidi, John Woods, Mohammed Al-Khalidi, Huosheng Hu \\ School of School of Computer Science and Electronic Engineering, University of Essex, CO4 3SQ, UK. \\ rjmohs@essex.ac.uk
}

\begin{abstract}
Current satellite communication remains very expensive and impractical for most small to mid-sized vessels, and at the same time marine wireless networking is lack of network coverage. To solve this problem, this paper proposes a novel IOT (Internet of Things) enabled system for marine data acquisition and cartography based on Ship Ad-hoc Networks (SANET's). Ships are equipped with Very High Frequency (VHF) radios and several sensors such as sea depth, temperature, wind speed and direction, etc. The collected sensory data is sent to $5 \mathrm{G}$ edge clouds incorporated at sink/base station nodes on shore, and ultimately aggregated at a central cloud on the internet to produce up to date cartography. The routing protocols deployed are DSDV (Destination-Sequenced Distance Vector), AODV (Ad hoc On-Demand Distance Vector), AOMDV (Ad hoc On-Demand Multipath Distance Vector) and DSR (Dynamic Source Routing) protocols, which are very popular in Mobile Ad-hoc Networks (MANET's) and compatible with multi hop routing environments and scalability towards increased traffic and mobility. Simulation results verify the feasibility and efficiency of the proposed system that has packet delivery rates of up to $80 \%$ at shore base stations.
\end{abstract}

Keywords: Internet of Things; Ship Ad-hoc Networks; Very High Frequency; On-demand Routing Schemes; Table Driven Routing Schemes; Marine Cartography Systems.

\section{Introduction}

The existing marine communication systems only provide low data rate services such as ship identification, positioning, etc. in the form of AIS (Automatic Identification System). Inter ship satellite communication is possible but is a costly option when compared to conventional wireless communications [1]. The existing AIS technology uses VHF radio frequencies to provide data such as location, course, heading, destination, tonnage, speed, etc. In preparation for the revolutionary $5 G$ networks, Ofcom has allocated more radio spectrum for the Internet of Things (IOT), specifically VHF spectrum aiming to encourage Machine to Machine (M2M) applications to use spectrum that will enable them to connect wirelessly over longer distances. This VHF spectrum has different properties to other frequencies, already in use for the IoT, and can reach distant locations which other frequencies may not.

Networking in the ship environment through Ship Ad Hoc Networks (SANET) is still in its infancy compared to other environments. On the other hand, VANETS (Vehicular Ad Hoc Networks) have received a lot of attention and have focused on using vehicles to form nodes in mobile Ad-Hoc networks. Anggoro et al. 
Rabab Al-Zaidi, John Woods, Mohammed Al-Khalidi, Huosheng Hu; An IOT-enabled System for Marine Data Acquisition and Cartography, Transactions on Networks and Communications, Volume 5 No. 1, February (2017); pp: 53-69

implemented Probabilistic Relay in OLSR and AODV routing protocols and compared their performance with the original protocols in a VANET [2]. With Probabilistic Relay they try to provide a reinforcement node to relay the data packet instead of waiting for retransmission when the data is dropped. Spaho et al. implemented two routing protocols: DSR and DYMO also in VANETs and investigated the performance of these routing protocols using PDR and throughput2 metrics [3]. They conclude that DYMO performs better than DSR. For the marine environment, Manoufali, et al. provided a detailed research study on maritime communication technologies to achieve reliable and resilient maritime wireless mesh networks [4]. Comprehensive guidelines were outlined to critically assess the different deployed maritime communications networks, and identify the milestones in the process of developing maritime wireless mesh networks (MWMNs). A method of producing a realistic model for constructing a VANET for shipping in a sea environment was presented in [5]. They gathered data from the international standard Automatic Identification System (AIS) used by commercial shipping and processed it to produce a realistic movement model. The model in an NS2 simulation was used to evaluate the application of MANET technologies to shipping. Two means of transmission rate control were proposed to maximize the system throughput subject to the reservation-based random access inherited from the Ad-Hoc self-organizing TDMA (ASOTDMA) protocol in a SANET. It was shown that the proposed control schemes could maintain the maximum system throughput adaptively as the number of ships varies for the different hop ranges and their target attempt rates.

Haider and Shabbir introduced a novel Genetic based approach for optimized routing Maritime Tactical Networks (MTN) and found the most efficient path having enough resources [6]. Optimized path selection criteria could maximize resources (bandwidth, battery power etc.), and any delay could be low and a path with a low hop count should be selected. Do et al. used a wireless Ad-Hoc network to provide an efficient positioning service and restores the weak sea-to-land communication link from small fishing boats to central base-stations [7]. The proposed network combines the Global Positioning System (GPS) service with a wireless Ad-Hoc network. The network simply utilizes the existing coastal radio network; therefore, greatly reducing cost and simplifying network design.

In this paper we extend our analysis and evaluation of the Ship Ad-hoc Network proposed in [8] [9] to IOT networks. A model of the VHF radio is used to setup a physical layer in the NS2 simulator, and four different routing protocols in mobile Ad-Hoc networks are used in simulation, namely DSDV, AODV, AOMDV and DSR. The performance of these protocols are analysed and compared in our marine environment where there is a high density in some regions (near ports) and sparse in others (open ocean).

The rest of this paper is structured as follows. Section 2 describes some background information about marine communications such as marine radio transmission and MANET Routing Protocols in the Marine Environment. Section 3 explains the node density and mobility characteristics in SANETs. A novel IOTenabled System for Marine Data Acquisition and Cartography is presented in Section 4. Section 5 briefly describes the implementation of the proposed system, including three examples. Experimental results are presented in Section 6 to demonstrate the feasibility and performance of the proposed system, including a brief comparison. Finally, the conclusion is given in Section 7. 


\section{Background}

\subsection{Marine Radio Transmission}

Very High Frequency (VHF) describes a particular part of the radio spectrum allocated to boats in a range of $156 \mathrm{MHz}-163 \mathrm{MHz}$ [10], namely Marine-band VHF radio which provides a means for vessels to communicate with each other and between shore stations (e.g. ports, locks, bridges and marinas) about operational, navigation and safety matters. A range of VHF radio channels exists, with specific3 permitted use in each case; for example, the channels allocated to port operations and ship movement may only be used on matters concerning navigation, vessel operations and safety. Other channels are allocated for ship to ship use, safety transmissions and contacting marinas. The system can use up to 25 watts and is effective over distances up to $100 \mathrm{~km}$ at sea in ideal circumstances.

Range is dependent on the height of the antenna used and atmospheric conditions. Range on land is often much less as a result of terrain, buildings and trees [10].

Automatic Identification System (AIS) is a VHF radio broadcasting system that transfers packets of data over VDL (VHF Data Link) and enables vessels and shore-based stations to send and receive identification information [11]. This information can help in location awareness, collision avoidance and provide an aid to navigation, by displaying emergency buoys and beacons. AIS can automatically issue static information (ship name, length, breadth, ship types, etc), dynamic information (real-time position, speed, course, heading, navigation state, etc.) and voyage information (draft, port of destination, estimated time of arrival, etc.). And also automatically receive this information from neighbouring ships. The IMO (International Maritime Organization) requires AIS to be installed aboard all ships of 300 gross tonnes and upwards engaged in international voyages, cargo ships of 500 gross tonnes and upwards not engaged in international voyages and all passenger ships irrespective of size [13]. Figure 1 shows a SANET over VHF physical links.

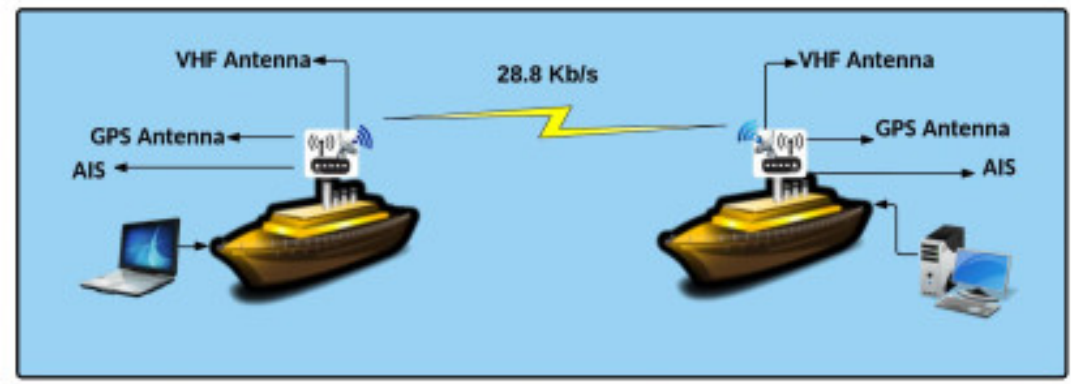

\subsection{MANET Routing Protocols in the Marine Environment}

The routing problem is one of the main difficulties in MANETs and also inherent in SANETs due to their mobile nature. Topologies can change due to node movement, radio interference and network partitions [14]. Many routing protocols have been proposed to deal with the routing challenges and the most popular of these protocols are:

(1) Ad hoc On-Demand Distance Vector Protocol (AODV) is an on-demand (reactive) routing protocol that uses a backward learning procedure in order to record the previous hop in the routing table. A number of enhanced approaches have been proposed to reduce the large overhead and high latency which result when encountering high packet loss in the AODV routing protocol [15]. Route 
Rabab Al-Zaidi, John Woods, Mohammed Al-Khalidi, Huosheng Hu; An IOT-enabled System for Marine Data Acquisition and Cartography, Transactions on Networks and Communications, Volume 5 No. 1, February (2017); pp: 53-69

discovery procedures are used by nodes to obtain routes on an as needed basis. RREQ duplicate copies are immediately discarded upon reception at every node. On receiving the first copy of a RREQ packet, the destination forms a reverse path in the same way as the intermediate nodes, it unicasts the RREP back to the source along the reverse path. As the RREP moves towards the source, it sets up a forward path to the destination at each hop [16].

(2) Ad hoc On-Demand Multipath Distance Vector Protocol (AOMDV) is an AODV protocol extension for providing multiple loop-free and link disjoint paths [17]. It can be utilized to find node-disjoint or link-disjoint routes. To find node-disjoint routes, every node does not reject duplicate RREQs immediately. Each RREQs that arrives through a different neighbor defines a node-disjoint path. This is because nodes cannot broadcast duplicate RREQs, therefore any two RREQs arriving at an intermediate node via a different neighbour of the same source could not have passed through the same node. After the first hop, the RREPs traverse the reverse paths, which are node disjoint and thus link-disjoint. The trajectories of every RREP may intersect at an intermediate node, but still each takes a different reverse path to the source to ensure link being disjoint [18].

(3) Dynamic Source Routing Protocol (DSR) is specifically designed for multi hop wireless Ad hoc networks. It is a simple and highly efficient Routing Protocol. The protocol is adaptive to the dynamic changes of the network topology by obtaining redundant routing information and fast response services [19]. The RREQ message contains the unique request id and records listing of the address for each of the intermediate nodes [20]. Each node transmitting the packets confirms the packets are received by the next node through the source route. If a confirmation is not received, this node sends a Route ERRor (RERR) message to the source node, here the link is broken [21].

(4) Destination-Sequenced Distance Vector Protocol (DSDV) is a hop-by-hop distance vector proactive routing protocol. Each node in the network maintains a routing table containing the next-hop, and the number of hops to all reachable destinations. To maintain the coherence of routing tables in a dynamic topology, each node periodically transmits updates, and also transmits updates instantly when important new information is available [22]. When a node along a path detects a broken route to a destination, it advertises its route to that destination with an infinite hop-count and a sequence number increased by one [23]. Whenever the network topology changes, a new sequence number is necessary before the network changes; therefore, DSDV is not suitable for highly dynamic networks [24].

\section{Node Density and Mobility Characteristics in SANETs}

\subsection{Node Density}

The number of nodes in the network (node density) and their movement patterns (node mobility) significantly affect the performance of the network. Route establishment in a sparse scattered network is usually difficult due to divisive communication ranges while a dense network may cause increased interference among the network nodes. High mobility rates may induce frequent link breakages leading to packet drops and delays in route establishments [25].

In reality, large mobile networks do not have uniform sets of nodes populated, and the number of node groups may be scattered around the network area. This situation can be found in the normal mobile network environment or other environments such as disaster areas, vehicular networks and others. 
Therefore, the constraints mentioned previously should be considered in evaluating the performance and scalability of MANETs and their routing protocols [26]. Node density in MANETs usually influences network connectivity and routing process as follows.

\subsubsection{Network Connectivity Rate}

The quality of network connectivity is key for improving the throughput of the network and has a big influence on the performance of routing protocol [27]. Network connectivity determination is based on the density of the number of neighbouring nodes. The density is defined according to the transmission range of the nodes. The connectivity density of a network is defined in [28][29].

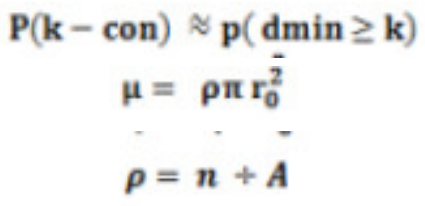

where $P$ is the probability of the connectivity; $n$ is the number of nodes located in the area; $d$ is the number of neighbours surrounding a node; $d_{\min }$ is the minimum degree of nodes; $k$ is mutually independent paths; is the density; represents the circumference; and $r$ is the radius of the transmission:

Based on this the criteria for determining the size of each "square" in the topology is known. The value of $\mathrm{k}$ is set to 1 , this means that in any particular network mentioned as dense given the probability of the connection of $P(k$-con $) \geq 0.95$ where $k=1$, there is 1 mutually independent path connecting the nodes in the particular network area. Thus the network can be categorized as (almost surely) 1-connected. This also implies that for any neighbours found to be within the transmission range of a particular node in the network, they are at most 2 hops away from each other. Figure 2 and Figure 3 show the scenario. The node density of the network areas will be based on the formulae provided for $\mathrm{P}(\mathrm{k}$-con). Therefore, an area can be considered dense when a MANET source node identifies that:

- It neighbours are at most 2 hops away from it and it has a mutually exclusive path to other neighbouring nodes that is independent of one another.

- $\quad \mathrm{P}(1$-con $) \geq 0.95$ Sparse areas are areas where nodes are isolated from a network or from one another:

- Nodes in a sparse neighbourhood cannot guarantee at least a single connection in the network (P (1-con) $\geq 0.95)$

- The minimal neighbour node degree for sparse areas could be $\mathrm{dmin}=0$. Thus, the node could be disconnected from the network. 


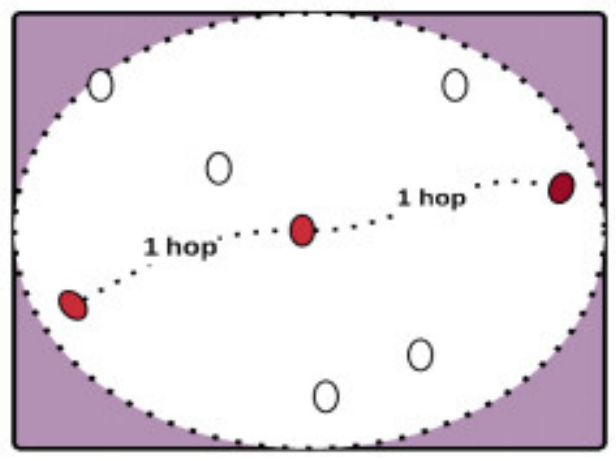

Figure $\mathbf{2}$ Node connecting to its neighbour located in the centre of the area

To solve existing problems in MANETs, many changes to the original routing protocols have been introduced. Some require small changes and some require large alterations of the original algorithms. In many cases MANET routing protocol alterations are related to the applications they are intended for. Whatever the intentions of the MANET, some common or basic problems still remain in the available protocols when they are moved into real implementations [26].

\subsubsection{Route Process}

Node density is also an important factor in the process of route selection and route repair. Increasing node density may bring more opportunities for selecting a route or repairing a route along with other benefits. If nodes are too crowded in the network, the hops between them increase the network overhead, and potentially cause the problem of load imbalance. Therefore, using a suitable node density within a realistic situation, network performance can be improved [27].

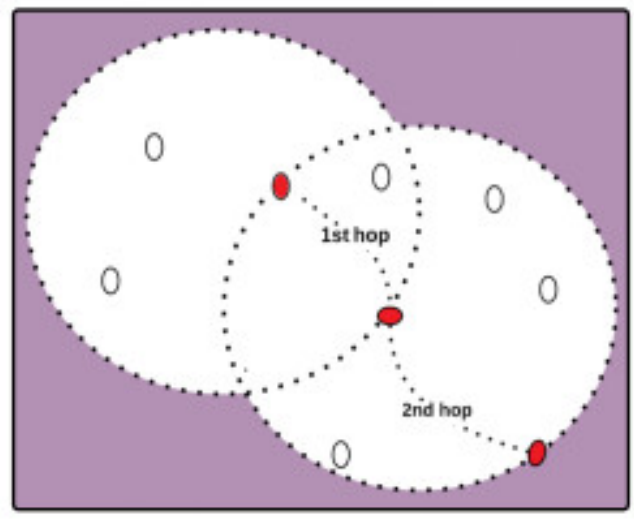

Figure 3 Node connecting to its neighbor not located at the center of the area

\subsection{Mobility Models}

Mobility models are designed to picture the movement patterns of mobile nodes, and describe how their location, velocity and accelerations change over time. Therefore, when evaluating MANET protocols, it is essential to choose the proper underlying mobility model [30]. Mobility modelling of nodes in Ad-Hoc networks is an immature science, because there are very few real-world Ad-Hoc networks with which models of node mobility can be compared [31]. Various mobility models have been categorised into several classes based on their specific mobility characteristics. For certain 
mobility models, the movement of a mobile node is likely to be affected by its movement history. These types of mobility models are referred to as having temporal dependency. In some mobility scenarios, the mobile nodes tend to move in a correlated manner. Such models are referred to as having spatial dependency. Another class is the mobility model with geographic restriction, where the movement of nodes is restricted by streets, freeways or obstacles. When simulating MANETs to measure the performance of various protocols, the majority of studies use the random waypoint mobility model [32]. While this mobility changes the network topology over time, it is often criticized as being unrealistic because actual node movement is not random in real life networks. Also due to the non-uniform node speed distribution, random waypoints show speed decay. Another issue is that the node movement has bias towards the centre of the simulation area which is as a result of the next destination selection by the moving nodes [33].

The mobility model is one of the key parameters which simulating a protocol depends on. Mobility models are designed to describe the movement patterns of mobile users, and how their location, velocity and acceleration changes over time. Since mobility patterns usually play a significant role in determining the protocol performance, it is necessary for mobility models to emulate the movement pattern of real life applications in a reasonable way. Otherwise, the observations and conclusions drawn from the simulation studies may be misleading. Therefore, when evaluating MANET protocols, it is essential to choose the proper underlying mobility model. For example, the nodes in the Random Waypoint model behave quite differently compared to nodes moving in groups [34]. It is not reasonable to evaluate the applications where nodes tend to move together using the Random Waypoint model. Therefore, there is a real need to develop a deeper understanding of mobility models and their impact on protocol performance [30]

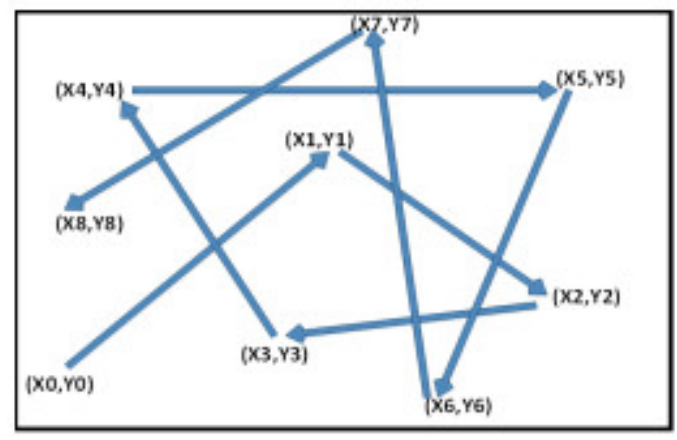

Figure 4 Node Movement example in the Random Waypoint Model

The Random Waypoint Mobility Model includes pause times that separate between changes in direction and/or speed. A mobile node stays in one location for a certain period of time (i.e., a pause time). Once this time expires, the mobile node chooses a random destination in the simulation area and a speed that is uniformly distributed between min-speed and max-speed. The mobile node then travels towards the newly chosen destination at the selected speed. When arriving, the mobile node pauses for a specified time period before starting the process again as seen in Figure 4 [35]. In the Random Waypoint model, Velocity (V) and Time (T) are the two main parameters that determine the mobility behaviour of nodes. If $\mathrm{V}$ is small and $\mathrm{T}$ is long, the topology of the Ad Hoc network becomes relatively stable. On the other hand, if the node moves fast ( $\mathrm{V}$ is large) and the pause time $\mathrm{T}$ is small, the topology is expected to be highly dynamic. Varying these two parameters, 


\section{pp: 53-69}

especially the $\mathrm{V}$ parameter, the Random Way point model can generate various mobility scenarios with different levels of nodal speed.

\section{The IOT-enabled Marine Data Acquisition and Cartography System}

A cartography application is proposed where the SANET is used to collect different marine sensory data from ships and vessels and send this data back to onshore sinks collocated with $5 \mathrm{G}$ base station's that include dedicated storage as part of the mobile edge computing (MEG) services. Mobile edge computing usually relates to mobile network applications and data stream acceleration through caching and/or compressing of relevant (mainly localized) data at the edge of the mobile network, as near as possible to the end user location.

In this paper, we propose a new application of MEC where part of the edge computing resources is exploited as edge repositories (clouds) of collected sensory data that successfully arrives to shore. The edge clouds eventually connect to a central cloud in the internet where all the sensory data is aggregated, filtered and analysed to produce real-time maps of surface and under water environmental information that produces accumulative maps for beneficiary customers. Figure 5 shows the proposed IOT-enabled system used for marine data acquisition and cartography. It can be used to collect data, including but not limited to: sea state, depth, temperature, wind speed/direction, humidity, salinity, etc.

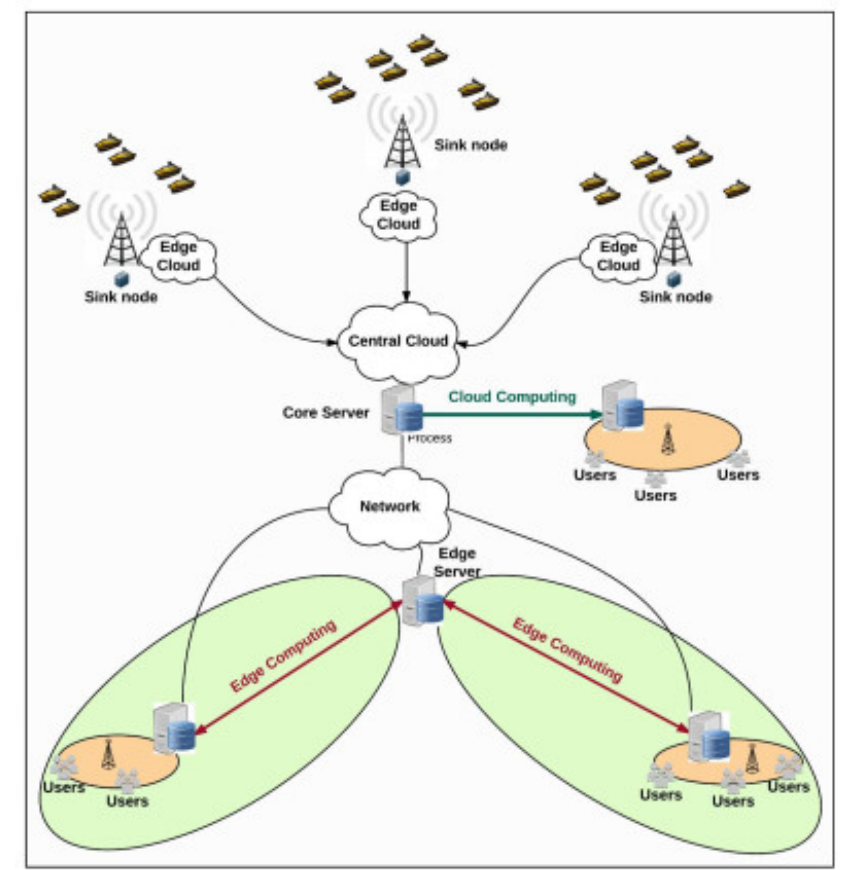

Figure 5 The proposed IOT-enabled Marine Data Acquisition and Cartography Systems

An identified obstacle is the bottleneck of traffic near the onshore sink. A quantization and compression method specific to marine sensory data has been proposed in our previous work [36] and partially alleviates this problem. For each of the sensors mentioned previously we have set the extreme lower and upper limits of the sensors readings likely to be found in the marine environment as well as the level of accuracy required to represent each reading. The predictability of gathered 
sensor data makes it beneficial to quantize the data to reduce the amount of bits needed to represent each reading in the binary representation. Applying this quantization in conjunction with the compression algorithm (AMDC) proposed in [36] has given effective data compression rates in comparison to the main compression methods.

\section{Implementation}

The network simulator NS2.35 is chosen for the implementation in this study, which runs under LINUX UBUNTU 12.04 LTS operating system. It is a discrete open source event simulator targeted at networking research. It provides substantial support for simulation of TCP, routing, and multicast protocols over wired and wireless (local and satellite) networks. It has a wide range of supported protocols and complex scenarios can be easily tested. NS2 is highly popular in the networking research community also due to its robustness and modularity. For simulating the routing protocols, the Tool Command Language (TCL) is used to initiate the event scheduler, set up the network topology, and tells traffic sources when to start and stop sending packets through the event scheduler. The final result is drawn from the execution of a TCL code written for this purpose. In order to extract the results from the trace files (.tr), we used an AWK script which is a powerful control command in LINUX that can process the rows and columns of the file to calculate the network performance metrics such as PDR, throughput, end to end delay, normalized routing load, etc.

\subsection{Physical Parameters}

To setup the marine VHF transmission environment, we input the actual transmission parameters into the physical layer of our nodes to achieve realistic modelling of the marine environment. The transmission frequency used is $156.975 \mathrm{MHZ}$ corresponding to channel 79 in the marine VHF band. The transmission power used is $25 \mathrm{Watts}$ which is used by most ships. The transmission and receive gain was set to 3 . The antenna height was set to 12 meters on average. We also use two transmission ranges in our simulation: one with transmission range of $30 \mathrm{Km}$ for some ships and another with $40 \mathrm{Km}$ for other ships [2]. The transmission ranges were calculated using the Free Space Propagation model below.

$$
p r=\frac{p t * G t * G r *(2)^{2}}{(4 \pi)^{2} * d^{2} * L}
$$

where $P$ t is the transmitted power, (d) is the received power, is the transmitter antenna gain, $G r$ is the receiver antenna gain, $d$ is the $T x-R x$ separation and $L$ is the system loss factor [36].

\subsection{Simulation Parameters}

The traffic source type used in the simulation is CBR (Constant Bit Rate) traffic. The mobility model used to generate node movement is the Random Way Point model in a different simulation. We used NS2(Network Simulator Version 2). The simulation was performed using four popular MANET routing protocols which are DSR, AODV, AOMDV and DSDV. For good resilience and accuracy, we have run the simulation 10 times and the average was extracted to analyse each performance factor for these four protocols. The number of CBR 
connections that were established in our simulation were 50 connections, the data packet size is 512 bits. Table 1 shows a summary of the parameters used in our simulation.

Table 1 Simulation Parameters

\begin{tabular}{|l|l|l|l|}
\hline \multicolumn{1}{|c|}{ Parameters } & Real Mobility in Dense Area & \multicolumn{1}{c|}{$\begin{array}{c}\text { Real Mobility in Sparse } \\
\text { Area }\end{array}$} & \multicolumn{1}{c|}{$\begin{array}{c}\text { Real Mobility in a Sparse } \\
\text { Area with Buoys }\end{array}$} \\
\hline Simulator & NS2.35 & NS2.35 & NS2.35 \\
\hline Routing Protocols & AODV,DSDV,AOMDV & AODV, DSDV, AOMDV & AODV, DSDV, AOMDV \\
\hline Simulation Time & $1000 \mathrm{sec}$ & $1000 \mathrm{sec}$ & $1000 \mathrm{sec}$ \\
\hline Traffic Type & CBR & CBR & CBR \\
\hline No of Nodes & 63 nodes with real locations & $\begin{array}{l}19 \text { nodes with real } \\
\text { locations }\end{array}$ & $\begin{array}{l}37 \text { nodes with real locations } \\
\text { with } 5,10,15,20 \text { buoys }\end{array}$ \\
\hline Simulation Area & $380 \mathrm{Km}$ X $150 \mathrm{Km}$ & $500 \mathrm{Km}$ X $400 \mathrm{Km}$ & $650 \mathrm{Km} \times 550 \mathrm{Km}$ \\
\hline Propagation Model & Free Space & Free Space & Free Space \\
\hline Transmission Range & $30 \mathrm{Km}, 40 \mathrm{Km}$ & $30 \mathrm{Km}, 40 \mathrm{Km}$ & $30 \mathrm{Km}, 40 \mathrm{Km}$ \\
\hline Movement Model & $\begin{array}{l}\text { Real mobility from live Als } \\
\text { website }\end{array}$ & $\begin{array}{l}\text { Real mobility from live } \\
\text { AIS website }\end{array}$ & $\begin{array}{l}\text { Real mobility from live AIS } \\
\text { website }\end{array}$ \\
\hline Bandwidth & $28.8 \mathrm{~Kb} / \mathrm{s}$ & $28.8 \mathrm{~Kb} / \mathrm{s}$ & $28.8 \mathrm{~Kb} / \mathrm{s}$ \\
\hline
\end{tabular}

\subsection{Experiment 1: Using Real Density and Mobility Patterns in a High Density} Area

This experiment is based on real ship locations, speed and direction in the English Channel also extracted from the AIS data website in [37] for cargo vessels, passenger vessels, tankers and fishing boats as shown in Figure 6. Parameters of this experiment have also been summarized in Table 1.

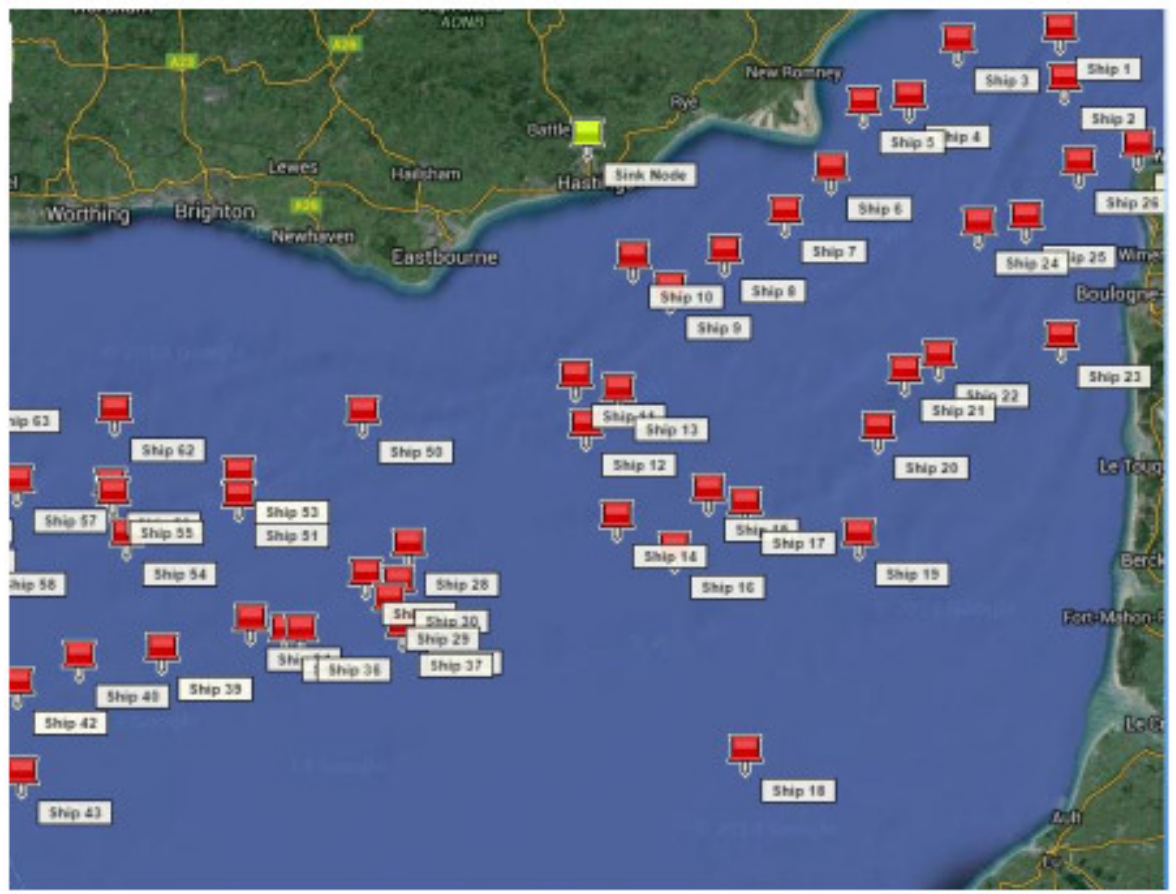

Figure 6 Real Ship Locations in English Channel 


\subsection{Experiment 2: Using Real Density and Mobility Patterns in a Low Density Area}

This experiment utilizes the vessel traffic in the North Sea as shown in Figure 7. Nineteen real mobile nodes have been simulated over area of $500 \mathrm{Km} \times 400 \mathrm{Km}$ extracted from the real AIS data website in [37] as in experiments 2 and 3. This experiment reflects a sparser network environment than the previous scenarios. The parameters used for this simulation are also shown in Table1.

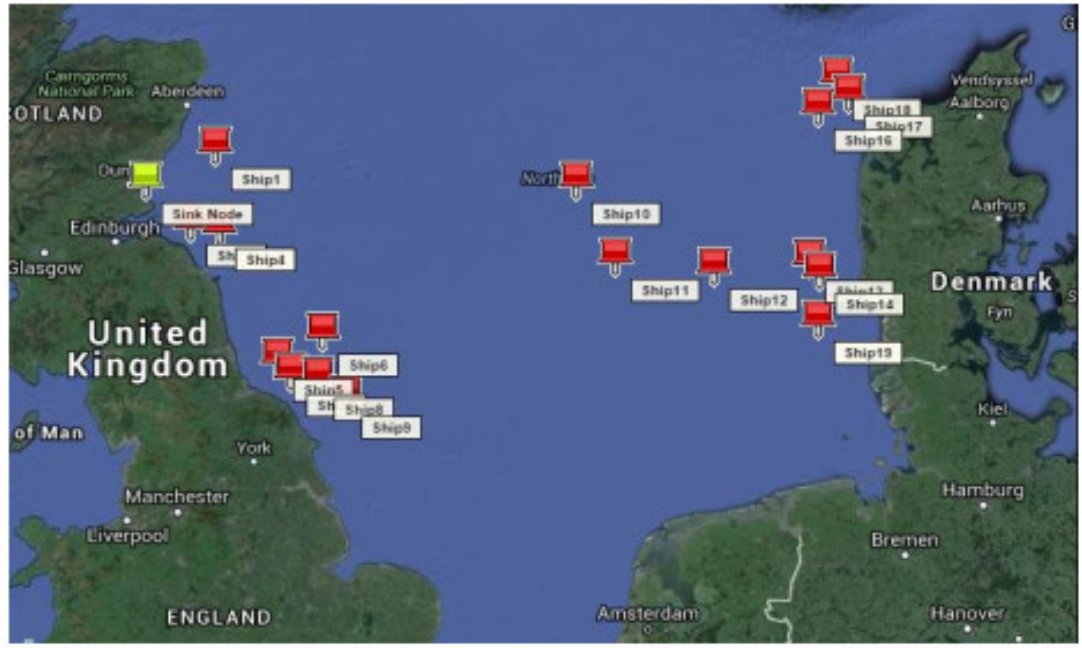

Figure 7 Real Ship Locations in the North Sea

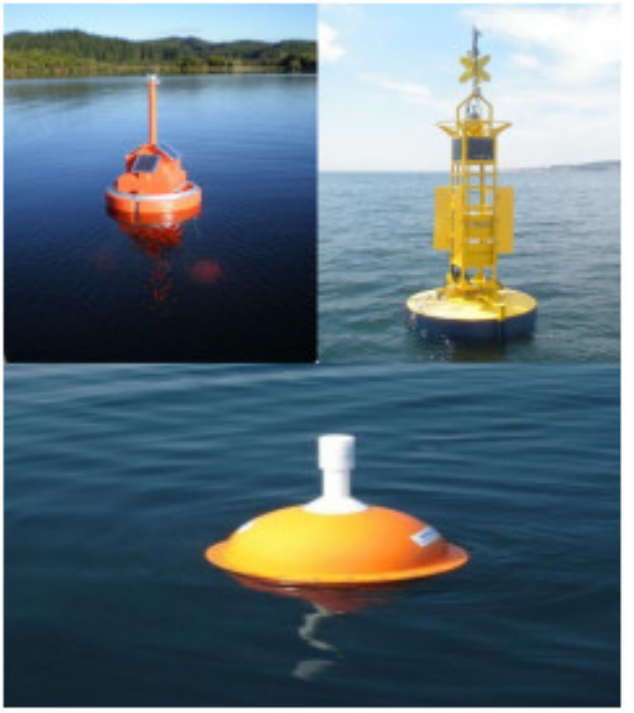

Figure 8 Types of Buoys

\subsection{Experiment 3: Using Marine Buoys to Reduce Isolated Sparse Areas}

Buoys are floating devices that can be anchored (stationary) or allowed to drift with ocean current. They are low power consumption devices that are usually solar cell powered and use energy harvesting schemes where energy is derived from solar power, captured, and stored for consumption. Buoys can be used as data collecting Systems for Meteorological/Oceanographic 


\section{pp: 53-69}

Parameters and can accommodate a selection of sensors depending on the application needs. Data from the Buoy can be transmitted real time and/or stored internally depending on network connectivity. There are differences in shapes and types as showed in Figure 8.

This experiment proposes the use of buoys to increase the density of forwarding nodes in sparse areas in order to increase the connectivity between ships. We use the buoys in experiment three with real location of ships for Iceland area as shown in Figure 9. The simulation was run with 5, 10, 15 and 20 buoys respectively located at highly disconnected locations. Table1 shows the parameters used in this experiment.

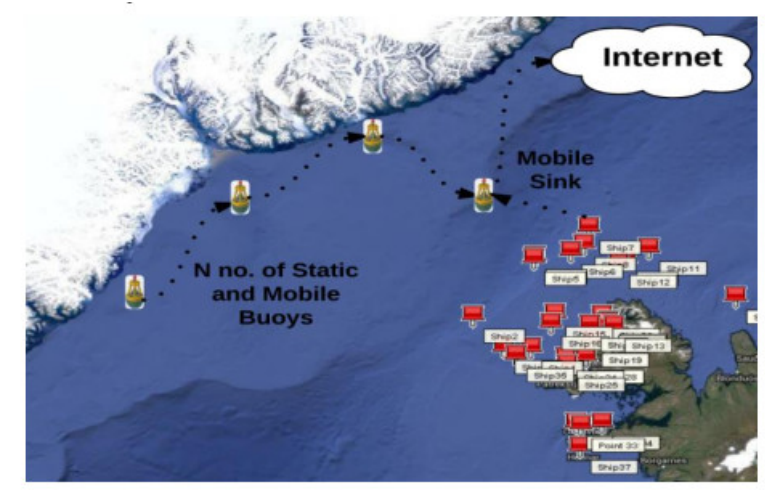

Figure 9 Real Ship Locations in the Iceland

\section{Results and Comparison}

Packet Delivery Ratio (PDR) is the metric used to evaluate the performance of MANET routing protocols in the marine environment. PDR is the ratio of successfully arrived data packets at the destination and can be calculated using

$$
P D R=\frac{\sum \text { Number of packet receive }}{\sum \text { Number of packet send }}
$$

In the first simulation scenario, when real mobility patterns in dense areas are applied to the nodes, high PDR rates are observed reaching a maximum of about $80 \%$ with AOMDV protocol and a minimum of $68 \%$ with DSDV protocol, as shown in Figure 10.

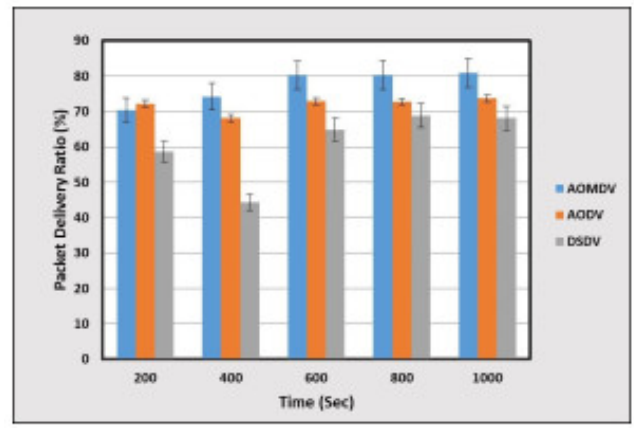

Figure 10 PDR of Real Density and Mobility in a Dense Area 
Figure 11 shows the effect of sparseness on the packet delivery ratio for the three protocols vs. time in the second simulation scenario. PDR for AOMDV protocol records the highest rate as in previous scenarios reaching about $40 \%$ while DSDV again records the lowest rate reaching approximately $27 \%$. Considering the VHF marine communication environment proposed and the performance of the routing protocols applied in this paper, it can be seen that the performance of MANETs in the marine environment decreases in low density situations where huge portions of sea have few vessels that can be used as multi hops to forward data packets. Therefore, to increase node density for a higher potential of PDR rates,

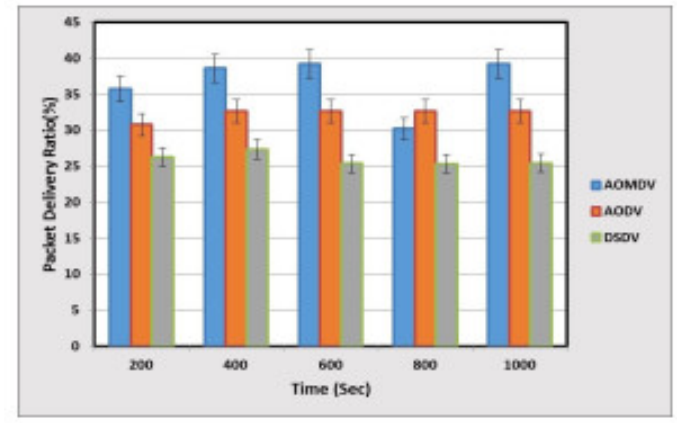

Figure 11 PDR of Real Density and Mobility in a Sparse Area

We have used buoys in our last simulation experiment which are distributed in the most disconnected areas of the water surfaces studied according to real maps of vessel tracks for a long period of time. Figure 12 shows the PDR rates vs. time according to distributing 5, 10, 15 and 20 buoys respectively. As can be seen from the figure, using 20 buoys to support the network connectivity increases PDR rates for the sparse area in Figure 11, reaching about 54\% for AOMDV.

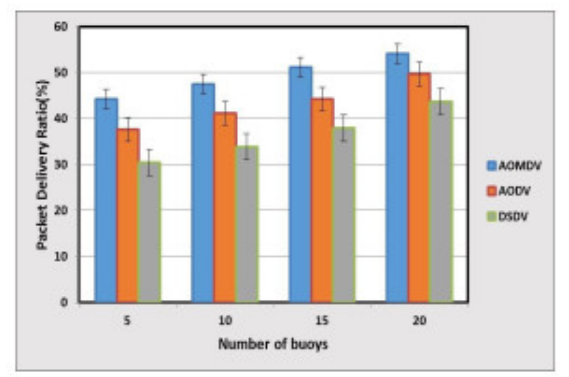

Figure 12 PDR of Real Density and Mobility in a Sparse Area with Buoys

\section{Conclusion}

This paper proposes a novel IOT-enabled system used for marine data acquisition and cartography. It explores the use of routing protocols to achieve a low cost AIS type system using existing on-board equipment. This infrastructure could use the existing VHF communication infrastructure available on ships and is effective for transmission ranges typically of $40 \mathrm{Km}$ in normal weather conditions carrying data traffic within the proposed network. The existing MANET protocols applied in this paper do have limitations in a marine environment, which can be sparse in some locations and dense in others. It can be concluded that AOMDV is the most efficient MANET for ship Ad-Hoc networks due to its multipath route discovery process, frequent link breakages in networks with high 
Rabab Al-Zaidi, John Woods, Mohammed Al-Khalidi, Huosheng Hu; An IOT-enabled System for Marine Data Acquisition and Cartography, Transactions on Networks and Communications, Volume 5 No. 1, February (2017); pp: 53-69

mobility rates, and maintaining alternative routes whenever needed. The source node and the intermediate nodes store the next-hop information corresponding to each flow for data packet transmission. Routes are established on demand and destination sequence numbers are used to find the latest route to destination. The connection setup delay is lower. A strong case can be made for the inclusion of store and forward on network nodes to make each ship retain packets as long as possible until another suitable candidate is available to pass the packet, which will be the subject of future investigation.

\section{REFERENCES}

[1] Hui, B.; Jeon, K.; Chang, K.; Kim, S.; Park, J.; Lim, Y. Design of radio transmission technologies for VHF band ship ad-hoc network. IEEE International Conference on ICT Convergence (ICTC), 2011, pp. 626-629.

[2] Anggoro, R.; Nakamura, R.; Kitasuka, T.; Itokawa, T.; Aritsugi, M. An evaluation of routing protocols with probabilistic relay in VANETs. IEEE, 2011, pp. 187-191.

[3] Spaho, E.; Ikeda, M.; Barolli, L.; Xhafa, F.; Kolici, V.; Takizawa, M. Performance analysis of DSR and DYMO routing protocols for VANETs. IEEE the 6th International Conference on Complex, Intelligent and Software Intensive Systems (CISIS), 2012, pp. 365-369.

[4] Manoufali, M.; Alshaer, H.; Kong, P.Y.; Jimaa, S. Technologies and networks supporting maritime wireless mesh communications. IEEE Wireless and Mobile Networking Conference (WMNC), 2013, pp. 1-8.15

[5] Pullin, A.J.; Presland, S.; Pattinson, C. Using Ship Movement in the Irish Sea for MANET Evaluation. Computer Modeling and Simulation. Second UKSIM European Symposium on EMS'08, 2008, pp. 394-399.

[6] Haider, Z.; Shabbir, F. Genetic based approach for optimized routing in Maritime Tactical MANETs. The 11th International Bhurban Conference on Applied Sciences and Technology (IBCAST), 2014, pp. 488-492.

[7] Do, D.; Nguyen, H.; Tran, N.; Ta, T.; Tran, T.; Vu, Y. Wireless ad hoc network based on global positioning system for marine monitoring, searching and rescuing (MSnR). Microwave Conference Proceedings (APMC), 2011 Asia-Pacific. IEEE, 2011, pp. 15101513.

[8] Mohsin, R.; Woods, J. Performance evaluation of MANET routing protocols in a maritime environment. IEEE Computer Science and Electronic Engineering Conference, 2014, pp. $1-5$. 
[9] Mohsin, R.J.; Woods, J.; Shawkat, M.Q. Density and mobility impact on MANET routing protocols in a maritime environment. IEEE Science and Information Conference (SAI), 2015, pp. 1046-1051.

[10] Pomfret, J. Guidelines on use of marine-Band VHF radio by INLand pleasure craft on UK freight water ways, 2013.

[11] Song, J.H.; Oh, K.R.; Kim, I.K.; Lee, J.Y. Application of maritime AIS (Automatic Identification System) to ADS-B (Automatic Dependent Surveillance. Control Automation and Systems (ICCAS), International Conference on, 2010, pp. 2233-2237.

[12] Zhu, F.; Lei, L. Research on a method for analysis of ship traffic density in harbor water area based on GIS. Conference Anthology, IEEE, 2013, pp. 1-4.

[13] Perkins, C.E.; Royer, E.M. Ad-hoc on-demand distance vector routing. Proceedings 2nd IEEE Workshop on Mobile Computing Systems and Applications,1999, pp. 90-100.

[14] Moravejosharieh, A.; Modares, H.; Salleh, R.; Mostajeran, E. Performance Analysis of AODV, AOMDV, DSR, DSDV Routing Protocols in Vehicular Ad Hoc Network. Research Journal of Recent Sciences ISSN 2013, 2277, 2502.

[15] Marina, M.K.; Das, S.R. Ad hoc on-demand multipath distance vector routing. Wireless Communications and Mobile Computing 2006, 6, 969-988.

[16] Biradar, S.; Majumder, K.; Sarkar, S.K.; Puttamadappa, C. Performance Evaluation and Comparison of AODV and AOMDV. International Journal on Computer Science \& Engineering 2010, 2.

[17] Broch, J.; Maltz, D.A.; Johnson, D.B.; Hu, Y.C.; Jetcheva, J. A performance comparison of multihop wireless ad hoc network routing protocols. Proceedings of the 4th annual ACM/IEEE international conference on Mobile computing and networking. ACM, 1998, pp. 85-97.

[18] Johnson, D.B.; Maltz, D.A. Dynamic source routing in ad hoc wireless networks. In Mobile computing; Springer, 1996; pp. 153-181.

[19] Maltz, D.B.J.D.A.; Broch, J. DSR: The dynamic source routing protocol for multi-hop wireless ad hoc networks. Computer Science Department, CMU, Pittsburgh, 2001, pp. 15213-3891.

[20] Kanthe, A.M.; Simunic, D.; Prasad, R. Comparison of AODV and DSR on-demand routing protocols in mobile ad hoc networks. Emerging Technology Trends in Electronics, The $1^{\text {st }}$ International Conference on Communication and Networking (ET2ECN), 2012, pp. 1-5. 
Rabab Al-Zaidi, John Woods, Mohammed Al-Khalidi, Huosheng Hu; An IOT-enabled System for Marine Data Acquisition and Cartography, Transactions on Networks and Communications, Volume 5 No. 1, February (2017);

[21] Johansson, P.; Larsson, T.; Hedman, N.; Mielczarek, B.; Degermark, M. Scenario-based performance analysis of routing protocols for mobile ad-hoc networks. Proceedings of the $5^{\text {th }}$ annual ACM/IEEE int. conf. on Mobile computing \& networking, 1999, pp. 195206.

[22] Parvathi, P. Comparative analysis of CBRP, AODV, DSDV routing protocols in mobile adhoc networks. International Conference on Computing, Communication and Applications (ICCCA), 2012, pp. 1-4.

[23] Usop, N.S.M.; Abdullah, A.; Abidin, A.F.A. Performance evaluation of AODV, DSDV \& DSR routing protocol in grid environment. International Journal of Computer Science and Network Security 2009, 9, 261-268.

[24] Thriveni, H.; Kumar, G.M.; Sharma, R. Performance Evaluation of Routing Protocols in Mobile Ad-Hoc Networks with Varying Node Density and Node Mobility. Communication Systems and Network Technologies (CSNT), 2013 International Conference on. IEEE, 2013, pp. 252-256.

[25] Natsheh, E.; Buragga, K. Nodes density and broadcast management in heterogeneous environments of mobile ad-hoc networks. Journal of Computer Science 2010, 6, 312.

[26] Zuo, J.; Wang, Y.; Liu, Y.; Zhang, Y. Performance evaluation of routing protocol in VANET with vehicle-node density. Wireless Communications Networking and Mobile Computing (WiCOM), 2010 6th International Conference on. IEEE, 2010, pp. 1-4.

[27] Bettstetter, C.; Zangl, J. How to achieve a connected ad hoc network with homogeneous range assignment: an analytical study with consideration of border effects. Mobile and Wireless Communications Network, 2002. 4th International Workshop on. IEEE, 2002, pp. $125-129$.

[28] Bettstetter, C. On the connectivity of wireless multi-hop networks with homogeneous and inhomogeneous range assignment. Proceedings of IEEE 56th Vehicular Technology Conference, 2002, Vol. 3, pp. 1706-1710.

[29] Bai, F.; Helmy, A. A survey of mobility models. Wireless Adhoc Networks. University of Southern California, USA 2004, 206.

[30] Raymond, D.; Burbey, I.; Zhao, Y.; Midkiff, S.; Koelling, C.P. Impact of Mobility Models on Simulated Ad Hoc Network Performance. Proc. 9th International Symposium on Wireless Personal Multimedia Communications (WPMC), 2006, pp. 398-402.

[31] Yoon, J.; Liu, M.; Noble, B. Random waypoint considered harmful. INFOCOM 2003. The $22^{\text {nd }}$ annual joint conference on computer and communications, 2003, Vol. 2, pp. 13121321. 
[32] Amjad, K.; Stocker, A.J. Impact of node density and mobility on the performance of AODV and DSR in MANETS. The 7th International Symposium on Communication Systems Networks and Digital Signal Processing (CSNDSP), 2010, pp. 61-65.

[33] Bai, F.; Sadagopan, N.; Helmy, A. IMPORTANT: A framework to systematically analyze the Impact of Mobility on Performance of RouTing protocols for Adhoc NeTworks. The 22 ${ }^{\text {nd }}$ Annual Joint Conference on Computer \& Communications. 2003, Vol. 2, pp. 825835.

[34] Camp, T.; Boleng, J.; Davies, V. A survey of mobility models for ad hoc network research. Wireless communications and mobile computing 2002, 2, 483-502.

[35] Mitra, A. Lecture Notes on Mobile Communication. A Curriculum Development Cell project Under QIP, IIT Guwahati 2009.

[36] Rabab J., M.; John, W.; Shawkat, M.Q. (AMDC) Algorithm for wireless sensor networks in the marine environment. Journal of Advanced Computer Science and Applications, $2015,6$.

[37] Marine Traffic. https://www.marinetraffic.com/en/ais/home/centerx:12/centery:25/zoom:4 